Reihe Rechtswissenschaft

Band 173 


\section{Die Spaltung von Aktiengesellschaften zwecks Privatisierung im Vergleich Deutschland - Frankreich}

Christophe Jäger

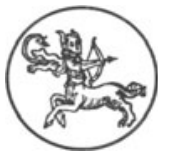

Centaurus Verlag \& Media UG 1995 


\section{Die Deutsche Bibliothek - CIP-Einheitsaufnahme}

\section{Jäger, Christophe:}

Die Spaltung von Aktiengesellschaften

zwecks Privatisierung im Vergleich

Deutschland - Frankreich / Christophe Jäger. -

Pfaffenweiler : Centaurus Verl.-Ges., 1995

(Reihe Rechtswissenschaft ; 173)

Zugl.: Freiburg (Breisgau), Univ., Diss., 1994

ISBN 978-3-89085-937-8 ISBN 978-3-86226-378-3 (eBook)

DOI 10.1007/978-3-86226-378-3

NE: GT

\section{ISSN 0177-2805}

Alle Rechte, insbesondere das Recht der Vervielfältigung und Verbreitung sowie der Übersetzung, vorbehalten. Kein Teil des Werkes darf in irgendeiner Form (durch Fotokopie, Mikrofilm oder ein anderes Verfahren) ohne schriftliche Genehmigung des Verlages reproduziert oder unter Verwendung elektronischer Systeme verarbeitet, vervielfältigt oder verbreitet werden.

(C) CENTAURUS-Verlagsgesellschaft mit beschränkter Haftung, Pfaffenweiler 1995

Satz: Vorlage des Autors 


\section{Vorwort}

Die vorliegende Arbeit hat im Wintersemester 1993/94 der Juristischen Fakultät der Albert-Ludwigs-Universität Freiburg als Dissertation vorgelegen. Für die Drucklegung wurden Rechtsprechung und Literatur bis Juli 1994 eingearbeitet. $\mathrm{Zu}$ diesem Zeitpunkt lagen für die Rechtslage de lege ferrenda der Diskussions- und der Referentenentwurf eines Gesetzes zur Bereinigung des Umwandlungsrechtes vor.

Frau Prof. Dr. Christine Windbichler, die die Arbeit angeregt und betreut hat, bin ich zu besonderem Dank verpflichtet. Ihre Beratung und Gesprächsbereitschaft waren mir stets eine wichtige Hilfe in meiner Arbeit. Herzlicher Dank gilt auch Herrn Prof. Dr. Fritz Rittner für die Erstellung des Zweitgutachtens, Herrn Prof. Dr. Dr. h.c. Manfred Löwisch für die Abfassung des Drittgutachtens und Herrn Prof. Dr. Meinrad Dreher für seine wertvollen Anregungen und hilfreichen Gespräche.

Schließlich möchte ich mich auch bei Frau Rechtsreferendarin Ute Melcher und den Herren Rechtsreferendaren Karl-Friedrich Dumoulin und Clemens Pfister bedanken, die mir bei der Durchsicht der Arbeit eine wertvolle Hilfe waren.

Freiburg, im Juli 1994

Christophe Jäger 


\section{INHALTSVERZEICHNIS}

Einleitung 1

Erster Teil: Spaltungen in Deutschland 6

\section{A) Rechtslage nach heutigem Recht außerhalb der Treuhandtätigkeit}

I) Grundlagen

1) Begriff der Spaltung 6

a) Aufspaltung 6

b) Abspaltung 7

c) Ausgliederung $\quad 7$

2) Begriff des staatlichen Unternehmens $\quad 8$

$\begin{array}{ll}\text { II) Fälle aus der Praxis } & 10\end{array}$

1) Beispiele für Aufspaltungen 10

2) Beispiele für Abspaltungen 10

3) Beispiele für Ausgliederungen 11

III) Verfahren bei Spaltungen nach heutigem Recht (außerhalb der Treuhandtätigkeit) 12

1) Verfahren bei der Aufspaltung 12

a) Beschluß über die Übertragung des Gesellschaftsvermögens auf andere Gesellschaften $\quad 12$

b) Beschluß über die Gründung der Nachfolgegesellschaften

c) Beschluß der Hauptversammlung über die Auflösung des übertragenden Unternehmens $\quad 14$

2) Verfahren bei der Abspaltung 15

a) Beschluß der Hauptversammlung über die Aus-
gründung zum Zwecke der Unternehmensteilung

aa) Mitwirkungspflicht der Hauptversammlung 15

bb) Erforderliche Mehrheit für einen solchen Beschluß

b) Gründung der neuen Nachfolgegesellschaft(en) 
c) Beschluß der Hauptversammlung über die Kapitalherabsetzung beim übertragenden Unternehmen

3) Verfahren bei der Ausgliederung 20

a) Analoge Anwendung des $\S 361$ Abs. $1 \mathrm{~S} .1 \mathrm{AktG}$

b) Beschluß nach $\S 119$ Abs. 2 AktG 20

c) Erfordernis einer satzungsmäßigen Ermächtigung bei der Ausgliederung $\quad 21$

d) Gründung der neuen Gesellschaft(en) 23

4) Ubergang des Gesellschaftsvermögens auf die

a) Bei noch zu gründenden Nachfolge-
gesellschaften

b) Bei schon bestehenden Nachfolge-
gesellschaften

5) Beteiligungsverhältnisse im Rahmen des

6) Möglichkeiten der Anfechtung von

Hauptversammlungsbeschlüssen im Rahmen des Spaltungsverfahrens

IV) Gläubigerschutz nach heutigem Recht (außerhalb der Treuhandtätigkeit)

1) Gläubigerschutz aus liquidationsrechtlichen Vorschriften

2) Gläubigerschutz aus den Vorschriften betreffend die Kapitalherabsetzung

3) Haftung der Nachfolgegesellschaften aus § 25 HGB 30

V) Arbeitnehmerschutz im Rahmen einer Spaltung 31

VI) Unterschiede zwischen der Spaltung zwecks

Privatisierung und der "herkömmlichen" Spaltung -

Besonderheiten der Privatisierung

\section{B) Die Spaltung zum Zwecke der Privatisierung durch die Treuhandanstalt im geltenden Recht}

I) Einführung $\quad 35$

II) Beispiele aus der Praxis $\quad 37$

1) Stamag-AG

2) Tridelta-AG

III) Arten von Spaltungen 38 
1) Verschiedene Stufen des Verfahrens 38

a) Spaltungsplan 39

b) Spaltungsbeschluß $\quad 41$

c) Anmeldung und Eintragung des Spaltungs-
beschlusses

d) Anmeldung des Spaltungsvorhabens und
Eintragung der neuen Gesellschaften

e) Spaltungsbericht und Prüfung der Spaltung 45

f) Auflösung der übertragenden Gesellschaft
im Falle der Aufspaltung

2) Übertragung des Gesellschaftsvermögens auf die
Nachfolgegesellschaften

3) Anfechtungsmöglichkeiten der

Hauptversammlungsbeschlüsse im Rahmen des SpTrUG 49

$\begin{array}{ll}\text { V) Gläubigerschutz } & 50\end{array}$

VI) Arbeitnehmerschutz 52

1) Anwendung des § 613a BGB 52

2) Sozialpläne im Rahmen des

a) Vorliegen einer Betriebsänderung 56

b) Interessenausgleich und Sozialplan $\quad 57$

c) Problemstellung $\quad 58$

aa) Abschluß des Sozialplans vor der Spaltung 58

bb) Abschluß des Sozialplans nach

Spaltungsvollzug

VII) Unterschiede zwischen der Spaltung zwecks

Privatisierung und der "herkömmlichen" Spaltung

- Besonderheiten der Privatisierung $\quad 61$

1) Rolle der Hauptversammlung und des Vorstandes 61

2) Gläubiger- und Arbeitnehmerschutz 63

C) Rechtslage nach zukünftigem Recht in Deutschland 64

$\begin{array}{ll}\text { I) Arten von Spaltungen } & 65\end{array}$

II) Verfahren im Rahmen der Spaltung nach dem Diskussions- und Referentenentwurf des BMJ 65

1) Verschiedene Stufen des Spaltungsverfahrens 65 
a) Aufspaltung 66

aa) Aufspaltung zur Aufnahme $\quad 66$

bb) Aufspaltung zur Neugründung $\quad 66$

b) Abspaltung $\quad 67$

c) Ausgliederung $\quad 68$

2) Übergang des Gesellschaftsvermögens auf die
Nachfolgegesellschaften

3) Beteiligungsverhältnisse im Rahmen des
Spaltungsverfahrens

4) Anfechtungsmöglichkeiten durch private Aktionäre $\quad 74$

$\begin{array}{ll}\text { III) Gläubigerschutz } & 76\end{array}$

$\begin{array}{ll}\text { IV) Arbeitnehmerschutz } & 77\end{array}$

V) Unterschiede zwischen der Spaltung zwecks Privatisierung und der "herkömmlichen"

$\begin{array}{ll}\text { Spaltung - Besonderheiten der Privatisierung } & 78\end{array}$

1) Rolle der Hauptversammlung $\quad 78$

2) Gläubiger- und Arbeitnehmerschutz $\quad 79$

$\begin{array}{ll}\text { Zweiter Teil: Spaltungen in Frankreich } & 80\end{array}$

I) Einführung: Der Begriff der Spaltung und des

öffentlichen Unternehmens im französischen Recht $\quad 80$

1) Einführung $\quad 80$

2) Definition der Spaltung $\quad 82$

a) Aufspaltung $\quad 82$

b) Abspaltung $\quad 82$

c) Ausgliederung $\quad 85$

3) Begriff des öffentlichen Unternehmens im
französischen Recht

a) Eigengesellschaft $\quad 86$

b) gemischt-wirtschaftliche Unternehmen 86

II) Verfahren bei der Spaltung einer

Aktiengesellschaft nach französischem Recht $\quad 88$

1) Stufen des Spaltungsverfahrens $\quad 88$

a) Aufspaltung $\quad 88$

aa) Projet de scission (Spaltungsplan) und 
contrat de scission (Spaltungsvertrag)

bb) Spaltungsbeschlu $B \quad 89$

cc) Gründung der Nachfolgegesellschaften im Fall der Spaltung zur Neugründung

dd) Kapitalerhöhung in den Nachfolgegesellschaft bei der Spaltung zur Aufnahme $\quad 90$

ee) Auflösung der übertragenden Gesellschaft 91

ff) Eintragung in das Handelsregister 92

gg) Überprüfung des Spaltungsplans durch die
Commissaires aux comptes et aux apports

hh) Prüfung des Spaltungsplans durch die C.O.B. 93

b) Verfahren bei dem sog. apport partiel d'actif, also der Abspaltung und Ausgliederung im deutschen Sinne

2) Anfechtung der für die Spaltung

erforderlichen Hauptversammlungsbeschlüsse

3) Übergang des Gesellschaftsvermögens auf die Nachfolgegesellschaften

4) Beteiligungsverhältnisse im Rahmen des Spaltungsverfahrens nach französischem Recht

III) Gläubigerschutz $\quad 98$

1) Bei der scission 98

2) Bei dem apport partiel d'actif 100

IV) Arbeitnehmerschutz 101

1) Übergang der Arbeitsverhältnisse 101

2) Plan social im Rahmen einer scission 102

a) Gesetzliche Grundlage 102

b) Schutz durch Art. 385 des Gesetzes
vom 24. Juli 1966

V) Unterschiede, die sich nach französischem Recht aus der Besonderheit des Kontextes "Privatisierung" gegenüber einer herkömmlichen Spaltung ergeben

1) Rolle der Hauptversammlung und des Vorstandes 103

2) Anfechtungsmöglichkeiten durch einzelne Aktionäre 105

3) Gläubiger- und Arbeitnehmerschutz 106

VI) Gründe für das Fehlen praktischer Fälle einer scission zum Zwecke der Privatisierung in Frankreich 
Dritter Teil: Vergleich zwischen der Rechtslage in Deutschland und Frankreich

I) Vergleich der Spaltungsgrundsätze

in den beiden Ländern

1) Aufspaltung

2) Abspaltung

3) Ausgliederung

II) Vergleich des Spaltungsverfahrens in

Frankreich und Deutschland

1) Vorüberlegung

2) Das Problem der Mitwirkung der

Hauptversammlung bei der Abspaltung und

Ausgliederung

3) Übergang des Gesellschaftsvermögens

117

4) Anfechtungsmöglichkeiten von Beschlüssen

118

5) Beteiligungsverhältnisse an den

Nachfolgegesellschaften

III) Gläubigerschutz

IV) Arbeitnehmerschutz

1) Übergang der Arbeitsverhältnisse

123

2) Haftung für Sozialplansansprüche

V) Vergleich der Unterschiede, die sich aus der

Besonderheit einer Spaltung zwecks Privatisierung gegenüber einer "herkömmlichen" Spaltung

in Deutschland und Frankreich ergeben

1) Rolle der Hauptversammlung $\quad 125$

2) Rolle des Vorstandes $\quad 126$

3) "Gefahr" der Anfechtung durch einzelne Aktionäre 127

4) Gläubiger- und Arbeitnehmerschutz

B) Auswertung des Rechtsvergleichs

I) Vorzug der Gesamtrechtsnachfolge

II) Das Problem der Mitwirkung der Hauptversammlung:

Fehlt im französischen Recht eine dem Holzmüller- 
Urteil des Bundesgerichtshofs entprechende

gerichtliche Entscheidung?

III) Anfechtungsmöglichkeiten und ihre Rechtsfolgen 132

IV) Einordnung des "apport partiel d'actif" (Abspaltung) als "scission" (Aufspaltung)?

V) Gläubigerschutz 134

$\begin{array}{ll}\text { VI) Arbeitnehmerschutz } & 135\end{array}$

$\begin{array}{ll}\text { Conclusio } & 136\end{array}$

Resümee in französischer Sprache 139

$\begin{array}{ll}\text { Literaturverzeichnis } & 145\end{array}$ 


\section{Abkürzungsverzeichnis:}

\begin{tabular}{|c|c|}
\hline a.A. & anderer Ansicht \\
\hline a.a.O. & am angegebenen Ort \\
\hline Abs. & Absatz \\
\hline $\mathrm{AcP}$ & Archiv für die civilistische Praxis \\
\hline AktG & Aktiengesetz \\
\hline Anm. & Anmerkung \\
\hline ArbuR & Arbeit und Recht \\
\hline $\mathrm{AuA}$ & Arbeit und Arbeitsrecht \\
\hline Aufl. & Auflage \\
\hline BB & Betriebsberater \\
\hline Bd. & Band \\
\hline BetrVG & Betriebsverfassungsgesetz \\
\hline BGB & Bürgerliches Gesetzbuch \\
\hline BGH & Bundesgerichtshof \\
\hline BlStSozArbR & $\begin{array}{l}\text { Blätter für Steuern, } \\
\text { Sozialversicherung und Arbeitsrecht }\end{array}$ \\
\hline BMJ & Bundesministerium der Justiz \\
\hline BR-Drucks. & Bundesratsdrucksache \\
\hline Bull. & Bulletin \\
\hline Bull. civ. & $\begin{array}{l}\text { Bulletin des arrêts de la Cour de cassation rendu en } \\
\text { matière civile }\end{array}$ \\
\hline $\mathrm{BuW}$ & Betrieb und Wirtschaft \\
\hline bzw. & beziehungsweise \\
\hline C.C. & Code civil \\
\hline C.O.B. & Commmission d'opération des bourses \\
\hline Cass. civ. & Cour de cassation, chambre civile \\
\hline Cass. com. & Cour de cassation, chambre commerciale \\
\hline Cass. soc. & Cour de cassation, chambre sociale \\
\hline d.h. & das heißt \\
\hline DB & Der Betrieb \\
\hline ders. & derselbe \\
\hline
\end{tabular}


Die AG

dies.

DisE

Diss.

DM

DtZ

DWiR

Ed.

EuGH

Fasc.

FAZ

FF

FGG

Fn.

Gaz. Pal.

$\mathrm{GmbH}$

GmbHR

HGB

Hrsg., hrsg.

i.V.m.

J.C.P.

JuS

m. Anm.

m.w.N.

NJW

Nr.

NZA

RdA

$\operatorname{Rdnr}(n)$.

RefE

Rev. des soc.
Die Aktiengesellschaft

dieselben

Diskussionsentwurf eines Gesetzes

zur Bereinigung des Umwandlungsrechts

Dissertation

Deutsche Mark

Deutsch-Deutsche Rechtszeitschrift

Deutsche Zeitschrift für Wirtschaftsrecht

Edition

Europäischer Gerichtshof

Fascicule

Frankfurter Allgemeine Zeitung

Francs Français

Gesetz zur freiwilligen Gerichtsbarkeit

Fußnote

Gazette du Palais

Gesellschaft mit beschränkter Haftung

GmbH-Rundschau

Handelsgesetzbuch

Herausgeber, herausgegeben

in Verbindung mit

Jurisclasseur périodique, La semaine juridique

Juristische Schulung

mit Anmerkung

mit weiteren Nachweisen

Neue Juristische Wochenschrift

Nummer

Neue Zeitschrift für Arbeits- und Sozialrecht

Recht der Arbeit

Randnummer(n)

Referentenentwurf eines Gesetzes

zur Bereinigung des Umwandlungsrechts

Revue des sociétés 
Rev. trim. dr. com.

RIW

S.

s.o.

somm.

somm. com.

SpTrUG

TreuhG

u.a.

vgl.

VIZ

WM

WR

z.B.

ZGR

ZHR

ZIP

ZVglRWiss.
Revue trimestrielle de droit commercial

Recht der internationalen Wirtschaft

Seite; Satz

siehe oben

sommaire

sommaire commenté

Gesetz über die Spaltung der von der

Treuhandanstalt verwalteten Unternehmen

Gesetz zur Privatisierung und Reorganisation des

volkseigenen Vermögens (Treuhandgesetz)

unter anderem

vergleiche

Zeitschrift für Vermögens- und Investitionsrecht

Zeitschrift für Wirtschafts- und

Bankrecht, Wertpapiermitteilungen

Wirtschaftsrecht

zum Beispiel

Zeitschrift für Unternehmens- und

Gesellschaftsrecht

Zeitschrift für das gesamte

Handelsrecht und Wirtschaftsrecht

Zeitschrift für Wirtschaftsrecht

Zeitschrift für vergleichende Rechtswissenschaft 